\title{
A RE-BRIGHTENING OF THE RADIO NEBULA ASSOCIATED WITH THE 2004 DECEMBER 27 GIANT FLARE FROM SGR 1806-20
}

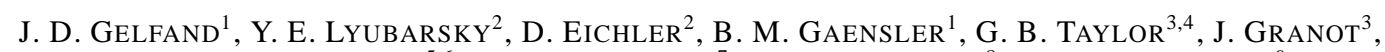 \\ K. J. Newton-MCGeE ${ }^{5,6}$, E. RAMirez-Ruiz ${ }^{7}$, C. Kouveliotou ${ }^{8}$, R. A. M. J. WiJers ${ }^{9}$ \\ Draft version March 13, 2005
}

\begin{abstract}
On 2004 Dec. 27, a giant $\gamma$-ray flare was detected from the magnetar SGR 1806-20. A radio observation seven days later revealed an expanding radio nebula at this position. Here we present results from an on-going monitoring campaign of this source with the Australia Telescope Compact Array and Very Large Array. These data indicate that there was an increase in the observed flux $\sim 25$ days after the initial $\gamma$-ray flare that lasted for $\sim 3-5$ days. In this Letter, we argue that this rebrightening marks the end of the coasting phase of the blast wave and the transition to the Sedov-Taylor phase. Assuming a distance to SGR 1806-20 of $15 \mathrm{kpc}$, we infer from the properties of this rebrightening that the blast wave is baryonic material of mass $M \gtrsim 10^{24.5} \mathrm{~g}$. In an accompanying paper (Granot et al. 2005), we show that this material was initially expanding with a velocity of about $0.4 c$; we therefore infer a kinetic energy $E \gtrsim 10^{44.5}$ ergs. If this mass was blown off the outer layers of the magnetar, it may have emitted a burst of ultra-high energy $(E>1 \mathrm{TeV})$ neutrinos far in excess of what might be expected from other astrophysical sources.
\end{abstract}

Subject headings: neutrinos - pulsars: individual (SGR 1806-20) — radio continuum: stars — shock waves stars: magnetic fields, neutron

\section{INTRODUCTION}

The soft gamma repeater (SGR) $1806-20$ is believed to be a magnetar - a slowly spinning neutron star with an extremely high magnetic field $\left(B \sim 10^{15}\right.$ gauss; Duncan \& Thompson 1992, Kouveliotou et al. 1998). On 2004 December 27, a giant flare of $\gamma$-rays was detected from this object (Borkowski et al. 2004), only the third such event detected from a magnetar. For a distance of $15 \mathrm{kpc}$ (Corbel \& Eikenberry 2004), the Dec. 27 flare was roughly a hundred times more luminous than the previous two such events (Palmer et al. 2005; Hurley et al. 2005 and references therein). Analysis of a Very Large Array (VLA) observation of SGR 1806-20 seven days after the flare discovered a bright, transient source, VLA J180839202439 (Cameron \& Kulkarni 2005; Gaensler et al. 2005a), believed to be a radio nebula driven by material blown off the magnetar during the flare. The detection of this source triggered a worldwide radio monitoring effort, the initial results of which have been presented by Gaensler et al. (2005c) and by Cameron et al. (2005). These observations determined that the radio source was expanding with constant velocity $\sim 0.4 c$ and that, after day 9, its flux decayed as a steep power law (Gaensler et al. 2005c; Taylor et al. in prep).

In this Letter, we present observational evidence for a shortterm rebrightening in the light curve. We argue this is the result of ambient material shocked by high-velocity material ejected from SGR 1806-20 during the Dec. 27 giant flare. In an accompanying paper (Granot et al. 2005), we expand the framework presented here and describe a full dynamical model of the in- teraction between the outflow ejected during the Dec. 27 giant flare and its surroundings.

This Letter is structured as follows: the observations are presented in $\S 2$, a theoretical model for this behavior is described in $\S 3$, a fit to the data using this model is carried out in $\S 4$, and its implications for the Dec. 27 burst are discussed in $§ 5$.

\section{OBSERVATIONS AND RESULTS}

As part of a long-term monitoring campaign of the radio nebula resulting from the Dec. 27 flare, we have observed SGR 1806-20 every few days at multiple frequencies with both the Australia Telescope Compact Array (ATCA) and with the VLA. Here we focus on observations at $4.8 \mathrm{GHz}$, as listed in Table 1. (Similar behavior is observed at other frequencies; a full multi-frequency study will be reported elsewhere.) The ATCA observations used a bandwidth of $128 \mathrm{MHz}$ at $4.8 \mathrm{GHz}$, and SGR 1806-20 was observed for $\sim 20$ minutes at this frequency in each observation. For each ATCA observation, we calibrated the flux density scale using an observation of PKS B1934-638 at the beginning of the run. We calibrated the phase at $4.8 \mathrm{GHz}$ using a short observation of PMN J1811-2055 taken approximately every three minutes during the observations. To minimize contamination from background emission in the field, we only used data from baselines that included the fixed antenna located $\sim 3 \mathrm{~km}$ away from the other five antennas of the array. The VLA observed SGR 1806-20 at $4.8 \mathrm{GHz}$ for $\sim 5-10 \mathrm{~min}-$ utes at each epoch using a total bandwidth of $100 \mathrm{MHz}$. These observations began with the VLA in its A configuration and

\footnotetext{
${ }^{1}$ Harvard-Smithsonian Center for Astrophysics, 60 Garden Street, Cambridge, MA 02138

${ }^{2}$ Department of Physics, Ben Gurion University, POB 653, Be'er Sheva 84105, Israel

${ }^{3}$ Kavli Institute for Particle Astrophysics and Cosmology, Stanford University, P.O. Box 20450, Stanford, CA 94309

${ }^{4}$ National Radio Astronomical Observatory, P.O. Box O, Socorro, NM 87801

${ }^{5}$ School of Physics, University of Sydney, NSW 2006, Australia

${ }^{6}$ Australia Telescope National Facility, CSIRO, P.O. Box 76, Epping, NSW 1710, Australia

${ }^{7}$ School of Natural Sciences, Institute for Advanced Study, Einstein Drive, Princeton, NJ 08540; Chandra Fellow

${ }^{8}$ NASA / Marshall Space Flight Center, NSSTC, XD-12, 320 Sparkman Drive, Huntsville, AL 35805

9 Astronomical Institute "Anton Pannekoek", University of Amsterdam, Kruislaan 403, 1098 SJ, Amsterdam, The Netherlands
} 
ended with the VLA in its B configuration. Absolute flux calibration was obtained from a short observation of 3C 286 during each run, and phase calibration was determined by observations of the calibrator PMN J1811-2055 every 4.5 minutes. For both the VLA and the ATCA observations, the radio flux density of SGR 1806-20 was measured using three different methods fitting the visibility data to a point source whose position was a free parameter, fitting the visibility data to a point source whose position is fixed at the location of the SGR, and measuring the peak brightness in an image made from these visibilities. In general, these three methods yielded consistent results, and any differences are reflected in the errors provided in Table 1.

The results from these observations are shown in Figure 1. As reported in Gaensler et al. (2005c), at day 9 there was a break in the light curve after which the radio flux faded rapidly. Starting on day 15, however, the observed flux from SGR 180620 began to deviate significantly from a power law decay, and on day 25 the flux began to increase for approximately five days. On day 31 , the observed flux began to decay again but at a slower rate than between days 9 and 15 . As argued in $\S 3$, we believe that this short-term rebrightening in the light curve is a result of the source's transition from the coasting phase to the Sedov-Taylor phase of its evolution.

\section{A SEMI-ANALYTIC MODEL}

In this Section, we present a semi-analytic model for the evolution of the radio source created during the Dec. 27 giant flare. We assume a spherical shell ${ }^{10}$ of mass $M$ expanding with an initial velocity $v_{0}$ into a medium of mass density $\rho$. Since this shell expands supersonically, it drives a forward shock into the ambient material. Initially, the newly swept up material is accumulated in a thin layer between the shell and the forward shock, and the equation of motion of the shell is:

$$
\frac{d}{d t}\left[\left(M+\frac{4 \pi}{3} R^{3} \rho\right) v\right]=4 \pi R^{2} p
$$

where $R=R(t)$ is the radius of the shell, $v=v(t)$ is the expansion velocity of the shell, and $p=p(t)$, the pressure inside of the shell, is found from energy conservation to be:

$$
\frac{1}{2} M v_{0}^{2}=\frac{1}{2}\left(M+\frac{4 \pi}{3} R^{3} \rho\right) v^{2}+2 \pi R^{3} p .
$$

This approximation also works well during the Sedov-Taylor phase (see Zeldovich \& Raizer 1966, and references therein), because even at this stage most of the swept-up material is accumulated in a thin layer just downstream of the shock whereas the rest of the volume is filled by a rarefied, hot gas at nearly constant pressure. By eliminating $p$ from the above equations and introducing dimensionless variables:

$$
\tau \equiv \frac{t}{t_{d e c}} ; r \equiv \frac{R}{v_{0} t_{d e c}} ; t_{d e c} \equiv\left[\left(\frac{4 \pi \rho}{3 M}\right)^{1 / 3} v_{0}\right]^{-1},
$$

one finds:

$$
\frac{d}{d \tau}\left[\left(1+r^{3}\right) \frac{d r}{d \tau}\right]=\frac{1}{r}\left[1-\left(1+r^{3}\right)\left(\frac{d r}{d \tau}\right)^{2}\right] .
$$

At $\tau \ll 1$, the solution to Equation (4) reduces to $v=v_{0}(1-$ $\left.0.8 r^{3}\right)$, while at $\tau \gg 1$, the solution to this equation asymptotically approaches $r=\left(2.5 \tau^{2}\right)^{1 / 5}$, close to the Sedov-Taylor solution.
We assume that, at the forward shock, electrons are heated to an energy $\gamma_{0} m_{e} c^{2}=\epsilon m_{p} v^{2}$, where $\epsilon$ is proportional to the fraction of the energy density behind the shock in relativistic electrons. ${ }^{11}$ Electrons with Lorentz factor $\gamma>\gamma_{0}$ are assumed to have a power-law energy spectrum $N(\gamma)=K\left(\gamma / \gamma_{0}\right)^{-p}$ (we assume $\gamma_{0}>1$, which is fulfilled for $\left.\epsilon>5 \times 10^{-4}[c / v]^{2}\right), N(\gamma) d \gamma$ is the number of electrons with energy between $\gamma m_{e} c^{2}$ and $(\gamma+d \gamma) m_{e} c^{2}, K=N\left(\gamma_{0}\right)$, and $p$ is the particle distribution index. Additionally, we assume that the magnetic energy density just downstream of the shock front is $B^{2} / 8 \pi=(9 / 8) \epsilon_{B} \rho v^{2}$, where $B$ is the magnetic field strength, and $\epsilon_{B}$ is the ratio of magnetic to internal energy density behind the shock. The observed radio spectrum corresponds to $p \approx 2.5$ (Gaensler et al. 2005c), a typical value for shock-accelerated electrons, and which we adopt in further discussion. If the number of emitting electrons is roughly $(4 \pi / 3) R^{2} v t \rho / m_{p}$, one can estimate the emission from the swept-up material as:

$$
S_{\nu}=a K(R / d)^{2} v t\left(\rho / m_{p}\right) \gamma_{0}^{p-1} B^{(1+p) / 2} \nu^{(1-p) / 2},
$$

where $d$ is the distance to the source, $S_{\nu}$ is the flux density at a frequency $\nu_{\mathrm{GHz}} \mathrm{GHz}$, and $a=4.7 \times 10^{-18}$ in cgs units. Substituting the quantities defined above into Equation (5), one obtains the synchrotron light curve:

$$
S_{\nu}(\tau)=11 \epsilon_{-1}^{1.5}\left(\epsilon_{B,-1} n_{-2}\right)^{0.87} M_{24} v_{10}^{4.75} d_{15}^{-2} \nu_{G H z}^{-0.75} f(\tau) m J y,
$$

where $d_{15}=d /(15 \mathrm{kpc})$, the ambient number density $n$ is defined as $n \equiv \rho / m_{p}$ and $n_{-2}=n /\left(0.01 \mathrm{~cm}^{-3}\right), \quad v_{10}=$ $v /\left(10^{10} \mathrm{~cm} \mathrm{~s}^{-1}\right), \epsilon_{-1}=\epsilon / 0.1, \epsilon_{B,-1}=\epsilon_{B} / 0.1$, and the dimensionless function $f(\tau)$ may be found from the solution $r(\tau)$ to Equation (4):

$$
f(\tau)=\tau r^{2}\left(\frac{d r}{d \tau}\right)^{(5 p-1) / 2} .
$$

Both $r(\tau)$ and $f(\tau)$ can be found from numerical integration of Equation (4), and are shown in Fig. 2. During the coasting phase $(\tau \ll 1)$, the luminosity grows as $t^{3}$ and reaches a maximum at $\tau=0.7$, at which point the expansion velocity has only decreased by $20 \%$. At $\tau \sim$ few, the luminosity decreases as $t^{-2.4}$; this is faster than the decrease during the Sedov-Taylor phase because the pressure within the cavity remains small for a long enough time and the expansion velocity decreases faster than in the Sedov-Taylor solution where the expanding envelope is filled by the hot gas. During the Sedov-Taylor phase ( $\tau \geq 10$ ), the luminosity declines as $t^{-1.65}$.

If the maximum flux is observed $30 t_{30}$ days after the explosion, one can estimate the mass and kinetic energy of the ejected material using Equation (3) to be:

$$
\begin{gathered}
M=3.2 n_{-2} t_{30}^{3} v_{10}^{3} \times 10^{24} \mathrm{~g}, \text { and } \\
E=\frac{1}{2} M v_{0}^{2}=1.6 n_{-2} t_{30}^{3} v_{10}^{5} \times 10^{44} \mathrm{ergs} .
\end{gathered}
$$

This estimate for the energy is strongly dependent on $v$, but fortunately not on projection effects. For $v \sim(0.15-0.6) c$, the actual velocity and that observed in projection on the sky are the same to within the experimental limits of the latter. That is, travel time effects, which give the illusion of a faster expansion, mostly cancel the geometric projection effects.

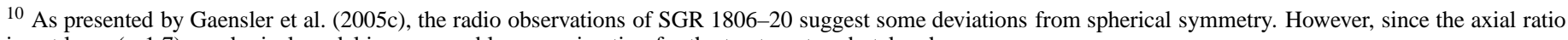
is not large $(\sim 1.7)$, a spherical model is a reasonable approximation for the treatment undertaken here.

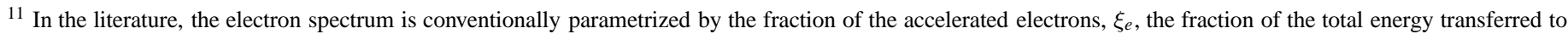
these electrons, $\epsilon_{e}$, and the particle distribution index $p$. In order to avoid cumbersome expressions, we introduce $\epsilon=\epsilon_{e}(p-2) / 2 \xi_{e}(p-1)$. 


\section{MODEL FITTING}

To determine the accuracy of the model presented in $\S 3$ and to use it to determine an alternate estimate of the initial mass and energy of the radio source, we fit the observed $4.8 \mathrm{GHz}$ flux densities after day 8.8 to the following model: ${ }^{12}$

$$
S_{\nu}(t)=S_{0}\left(\frac{t}{9 \text { days }}\right)^{\delta}+11 A \nu_{\mathrm{GHz}}^{-0.75} f\left(t / t_{\mathrm{dec}}\right) \mathrm{mJy} ;
$$

where $S_{0}$ mJy is the flux density on day $9, \delta$ is the index of the underlying power-law decay, and:

$$
A=\epsilon_{-1}^{1.5}\left(\epsilon_{B,-1} n_{-2}\right)^{0.87} M_{24} v_{10}^{4.75} d_{15}^{-2},
$$

as derived from Equation (6). The fit was performed using a $\chi^{2}$-minimizing gradient-expansion algorithm, and the bestfit parameters are $S_{0}=52.4 \pm 1.3 \mathrm{mJy}, \delta=-3.12 \pm 0.11, A=$ $11.9 \pm 0.2$, and $t_{\mathrm{dec}}=46.5 \pm 1.7$ days. The best-fit model (reduced $\chi^{2}=1.23$ ) is shown in Figure 1 . This model predicts that at $t \approx t_{d e c}$, a decrease in the source's expansion velocity should be observed. This possibility can be directly tested through VLA imaging of the source at $8.5 \mathrm{GHz}$, as will be discussed in a forthcoming paper (Taylor et al. in prep). The fact that the shape of the observed rebrightening deviates slightly from the prediction is not particularly surprising given the simplicity of the model described in $\S 3$, and could be due to a myriad of factors - e.g. a change in the ambient density as the source expands or turbulence in the layer of shocked ambient material. In a subsequent paper, we will present a more detailed model for the evolution of the observed source. However, the present fit is good enough that we can use the above values of $A$ and $t_{\mathrm{dec}}$ to directly express the ejected mass in terms of the unknowns $\epsilon, \epsilon_{B,-1}, n_{-2}, v_{10}$ and $d_{15}$. Rather than use the expressions for $A$ and $t_{\mathrm{dec}}$ to completely eliminate one of these quantities, we adopt an expression for $M$ which jointly minimizes the powerlaw dependences of all five parameters. We then find:

$$
M=6.6 \epsilon_{-1}^{-0.64} \epsilon_{B,-1}^{-0.37} n_{-2}^{0.20} v_{10}^{-0.32} d_{15}^{0.86} \times 10^{24} \mathrm{~g},
$$

which in turn implies

$$
E=3.3 \epsilon_{-1}^{-0.64} \epsilon_{B,-1}^{-0.37} n_{-2}^{0.20} v_{10}^{1.68} d_{15}^{0.86} \times 10^{44} \text { ergs. }
$$

Note that in these Equations, $M$ and $E$ are only weakly dependent on the ambient density, $n$ (which is a difficult quantity to constrain from observations), are somewhat more sensitive to the quantities that describe the shock physics $\left(\epsilon\right.$ and $\left.\epsilon_{B}\right)$. The total energetics of Equation (9) suggest that $n_{-2}<10^{3}$. For a distance of $15 \mathrm{kpc}$, a number density of $n_{-2} \approx 10$, and initial expansion velocity of $v_{10} \approx 1.2$ (the approximate initial expansion velocity of the source given by Taylor et al. in prep), the estimated initial mass is $M=1.1 \epsilon_{-1}^{-0.64} \epsilon_{B,-1}^{-0.37} \times 10^{25} \mathrm{~g}$. While $\epsilon$ and $\epsilon_{B}$ are still unknown quantities, there are estimates available from studies of gamma-ray bursts and supernova remnants. If the expanding nebula behaves like the relativistic jets produced in gamma-ray bursts (GRBs), then $\epsilon \sim 10^{-2.5}-10^{-1.5}$ and $\epsilon_{B} \sim 10^{-5}-10^{-1}$ (Panaitescu \& Kumar 2002), implying that $M \sim 10^{25} \mathrm{~g}$ for high $\epsilon_{B}$ and $M \sim 10^{27} \mathrm{~g}$ for low $\epsilon_{B}$. However, if the behavior of the expanding nebula is closer to that of a supernova blast-wave, the magnetic field and relativistic electrons will be in energy equipartition. In this case, $\epsilon_{B} \approx \epsilon$ (Bamba et al. 2003) and $\epsilon \sim 10^{-2}-10^{-3}$ (Ellison et al. 2000), so the inferred initial mass would be $M \sim 10^{26}-10^{27} \mathrm{~g}$.

It is possible that the density around SGR 1806-20 is considerably different from $n \approx 0.1 \mathrm{~cm}^{-3}$ as assumed above. In
Gaensler et al. (2005c), we claim that the nebula initially expanded into a low-density cavity carved out by the quiescent wind of SGR 1806-20. However, by the time of the rebrightening discussed here, the nebula has already expanded beyond this tiny cavity and is interacting with the surrounding medium. There is evidence that SGR 1806-20 is associated with the H II complex W31 (Corbel \& Eikenberry 2004), and may be located inside a cluster of massive stars embedded in a dust cloud (Fuchs et al. 1999). If the ejected material from SGR 1806-20 is expanding inside a stellar wind bubble formed by its progenitor (cf. Gaensler et al. 2005b) or by one of the nearby massive stars, then $n$ could be as low $\sim 10^{-3} \mathrm{~cm}^{-3}$. In this case, the values for $M$ estimated above are a factor of $\sim 2-3$ too high. However, if the ejected mass from SGR 1806-20 is expanding inside a dust cloud, then $n$ could be as high as $\sim 10 \mathrm{~cm}^{-3}$ if which case the values for $M$ estimated in the previous paragraph are a factor of $\sim 3-4$ too low. In either case, the uncertainty in the density around SGR 1806-20 does not change the order of magnitude of the ejected mass and energy. These estimates of $M$ and $E$ are similar to those in Equations (8) and (9), which depend on the peak in the light curve $\left(t_{30}\right)$ but are independent of the shock physics. As a result, we conclude that the Dec. 27 flare created a nebula with initial mass $\gtrsim 10^{24.5} \mathrm{~g}$ and initial kinetic energy $\gtrsim 10^{44.5}$ ergs.

\section{DISCUSSION}

An inherent assumption in the model presented in $\$ 3$ is that most of the energy of the radio source is in the form of modestly relativistic or sub-relativistic baryons. In an accompanying paper by Granot et al. (2005), we perform a more detailed analysis of the dynamics, starting at earlier times before the rebrightening in the light curve, and show that the outflow must indeed be mildly relativistic. This is consistent with the observational data, as presented by Taylor et al. (in prep).

A possible source of these baryons is the neutron star itself. If we assume that the energy of the flare, $\sim 10^{46} \mathrm{ergs}$, was released uniformly above and below the surface, and that the radius of the neutron star is $10^{6} \mathrm{~cm}$, then the Dec. 27 flare deposited an energy density of $10^{28} \mathrm{ergs} \mathrm{cm}^{-3}$ within SGR 1806-20, and matter less dense than $10^{8} \mathrm{~g} \mathrm{~cm}^{-3}$ will be ejected off the surface (Palmer et al. 2005). The outermost layers of a magnetar are typically less dense than this critical value (e.g. Lyubarsky et al. 2002 and references therein for the crust structure of magnetars), and we expect that roughly $0.3 \%$ of the star's volume will be blown off. As a result, $\sim 1 \%$ of the energy in the flare is in baryons, close to the inferred value (Gaensler et al. $2005 c$ ). Since the escape velocity of a neutron star is $\sim 0.3 c$, this mechanism for generating the radio nebula also explains the observed initial expansion velocity. However, if all of the inferred ejecta were released from the surface of the NS during the initial "hard spike" $(\lesssim 0.5 \mathrm{~s})$ of the giant flare, the outflow would be opaque to $\gamma$-rays and the Dec. 27 flare would not have been observed. This can be avoided if there are spots on the magnetar surface from which radiation is expelled without matter, and other points from which matter is expelled.

One possible observational signature of this process is the detection of ultra high-energy (UHE; $E_{\nu}>1 \mathrm{TeV}$ ) neutrinos from SGR 1806-20 coincident with the Dec. 27 flare. In this non-relativistic wind, internal shocks produced by significant

\footnotetext{
12 We only used data after day 8.8 in this fit because, as reported in Gaensler et al. (2005c), there is a break in the light curve at this epoch which cannot be explained
} by the model presented in $\$ 3$. 
variations in the outflow velocity within 0.5 light seconds of the star will accelerate some protons to energies high enough that they create pions through collisions with other protons. When these pions decay, they can produce $\mathrm{TeV}$ neutrinos. If the total energy in neutrinos is $\epsilon_{\nu} E$, where $E$ is the initial kinetic energy of the ejecta as estimated in Equations (9) and (13), then the observed fluence of neutrinos, $\mathcal{F}_{\nu}$, is:

$$
\mathcal{F}_{\nu} \approx 1.2 \epsilon_{\nu,-1} E_{44.5} d_{15}^{-2} \times 10^{-3} \mathrm{ergs} \mathrm{cm}^{-2}
$$

where $E_{44.5}=E / 10^{44.5}$ ergs and $\epsilon_{\nu,-1}=\epsilon_{\nu} / 0.1$ (e.g. Eichler \& Schramm 1978). This is much higher than the $10^{-5} \mathrm{erg} \mathrm{cm}^{-2}$ typically expected from bright GRBs (Eichler 1994). Depending on the exact values of $\epsilon_{\nu}$ and $E$, these neutrinos could possibly have been detected with current arrays, and the Dec. 27 event thus makes the best test case so far for testing the hypothesis of UHE neutrino emission from $\gamma$-ray outbursts.
The National Radio Astronomy Observatory is a facility of the National Science Foundation operated under cooperative agreement by Associated Universities, Inc. The Australia Telescope (ATCA) is funded by the Commonwealth of Australia for operation as a National Facility managed by CSIRO. We thank Bob Sault, Barry Clark, and Jean Wrobel for scheduling of the VLA and ATCA observations, and Roland Crocker and John Raymond for useful conversations. B.M.G. and J.D.G. acknowledge the support of NASA through a Long Term Space Astrophysics grant. D.E. acknowledges support from the Israel-U.S. BSF, the ISF, and the Arnow Chair of Physics. Y.L acknowledges support from the German-Israeli Foundation. E.R.R is sponsored by NASA through a Chandra Postdoctoral Fellowship award PF3-40028. R.A.M.J.W acknowledges support from NWO.

\section{REFERENCES}

Bamba et al. 2003, ApJ, 589, 827

Borkowski et al. 2004, GRB Circular Network, 2920

Cameron, P. B. \& Kulkarni, S. R. 2005, GRB Circular Network, 2928

Cameron et al. 2005, Nature submitted (astro-ph/0502428)

Corbel, S. \& Eikenberry, S. S. 2004, A\&A, 419, 191

Duncan, R. C. \& Thompson, C. 1992, ApJ, 392, L9

Eichler, D. 1994, ApJS, 90, 877

Eichler, D. \& Schramm, D. N. 1978, Nature, 275, 704

Ellison, D. C., Berezhko, E. G., \& Baring, M. G. 2000, ApJ, 540, 292

Fuchs et al. 1999, A\&A, 350, 891

Gaensler et al. 2005a, GRB Circular Network, 2929
Gaensler et al. 2005b, ApJ, 620, L95

Gaensler et al. 2005c, Nature, in press (astro-ph/0502393)

Granot et al. 2005, ApJL submitted (astro-ph/0503251)

Hurley et al. 2005, Nature submitted (astro-ph/0502329)

Kouveliotou et al. 1998, Nature, 393, 235

Lyubarsky, Y., Eichler, D., \& Thompson, C. 2002, ApJ, 580, L69

Palmer et al. 2005, Nature in press (astro-ph/0503030)

Panaitescu, A. \& Kumar, P. 2002, ApJ, 571, 779

Zeldovich, Y. \& Raizer, Y. P. 1966, Elements of gasdynamics and the classical theory of shock waves (New York: Academic Press, 1966, edited by Hayes, W.D.; Probstein, Ronald F.) 
TABLE 1

RADIO OBSERVATIONS AT 4.8 GHz OF THE RADIO NEBULA PRODUCED BY SGR 1806-20

\begin{tabular}{|c|c|c|c|}
\hline Average Epoch [UT] & Days after Burst & Telescope & $S_{4.8 \mathrm{GHz}}[\mathrm{mJy}]$ \\
\hline 2005 Jan 03.83 & 6.93 & VLA & $80 \pm 1$ \\
\hline 2005 Jan 04.61 & 7.71 & VLA & $66 \pm 3$ \\
\hline 2005 Jan 05.26 & 8.36 & ATCA & $60 \pm 1$ \\
\hline 2005 Jan 05.66 & 8.76 & VLA & $57 \pm 3$ \\
\hline 2005 Jan 05.85 & 8.95 & ATCA & $53 \pm 1$ \\
\hline 2005 Jan 06.24 & 9.34 & ATCA & $46 \pm 2$ \\
\hline $2005 \mathrm{Jan} 06.84$ & 9.94 & ATCA & $39 \pm 2$ \\
\hline 2005 Jan 06.84 & 9.94 & VLA & $39 \pm 1$ \\
\hline 2005 Jan 07.90 & 11.00 & VLA & $28 \pm 2$ \\
\hline 2005 Jan 08.19 & 11.29 & ATCA & $25 \pm 2$ \\
\hline 2005 Jan 09.07 & 12.17 & ATCA & $21 \pm 1$ \\
\hline 2005 Jan 10.07 & 13.17 & ATCA & $17 \pm 1$ \\
\hline 2005 Jan 12.06 & 15.16 & ATCA & $12 \pm 1$ \\
\hline 2005 Jan 14.08 & 17.18 & ATCA & $10 \pm 1$ \\
\hline 2005 Jan 16.08 & 19.18 & ATCA & $7 \pm 1$ \\
\hline 2005 Jan 18.01 & 21.11 & ATCA & $6.5 \pm 0.5$ \\
\hline 2005 Jan 20.01 & 23.11 & ATCA & $5.5 \pm 0.5$ \\
\hline $2005 \mathrm{Jan} 22.08$ & 25.18 & ATCA & $4.5 \pm 0.5$ \\
\hline 2005 Jan 23.08 & 26.18 & ATCA & $5.5 \pm 0.5$ \\
\hline 2005 Jan 24.62 & 27.72 & VLA & $5.0 \pm 0.2$ \\
\hline 2005 Jan 24.81 & 27.91 & ATCA & $4.4 \pm 0.5$ \\
\hline 2005 Jan 25.99 & 29.09 & ATCA & $5.5 \pm 0.5$ \\
\hline 2005 Jan 27.99 & 31.09 & ATCA & $5.8 \pm 0.5$ \\
\hline 2005 Jan 29.99 & 33.09 & ATCA & $5.5 \pm 0.5$ \\
\hline $2005 \mathrm{Jan} 31.82$ & 34.92 & ATCA & $6.0 \pm 0.5$ \\
\hline 2005 Feb 01.82 & 35.92 & ATCA & $5.2 \pm 0.3$ \\
\hline 2005 Feb 02.82 & 36.92 & ATCA & $5.8 \pm 0.4$ \\
\hline 2005 Feb 03.59 & 37.69 & VLA & $4.8 \pm 0.2$ \\
\hline 2005 Feb 03.82 & 37.92 & ATCA & $4.8 \pm 0.3$ \\
\hline 2005 Feb 05.91 & 40.01 & ATCA & $4.4 \pm 0.3$ \\
\hline 2005 Feb 07.53 & 41.63 & VLA & $4.1 \pm 0.2$ \\
\hline 2005 Feb 10.52 & 44.62 & VLA & $3.9 \pm 0.2$ \\
\hline 2005 Feb 11.92 & 46.02 & ATCA & $3.6 \pm 0.4$ \\
\hline 2005 Feb 12.62 & 46.72 & VLA & $4.2 \pm 0.2$ \\
\hline 2005 Feb 14.80 & 48.90 & ATCA & $3.5 \pm 0.2$ \\
\hline 2005 Feb 19.54 & 53.64 & VLA & $3.3 \pm 0.2$ \\
\hline 2005 Feb 20.98 & 55.08 & ATCA & $2.9 \pm 0.4$ \\
\hline 2005 Feb 21.61 & 55.71 & VLA & $3.3 \pm 0.1$ \\
\hline 2005 Feb 23.99 & 58.09 & ATCA & $2.8 \pm 0.5$ \\
\hline 2005 Feb 26.55 & 60.65 & VLA & $2.7 \pm 0.1$ \\
\hline $2005 \mathrm{Feb} 28.85$ & 62.95 & ATCA & $2.5 \pm 0.4$ \\
\hline
\end{tabular}

Note. - Flux densities before 2005 Jan 18.01 are also reported in the Supplementary Section of Gaensler et al. (2005c). 


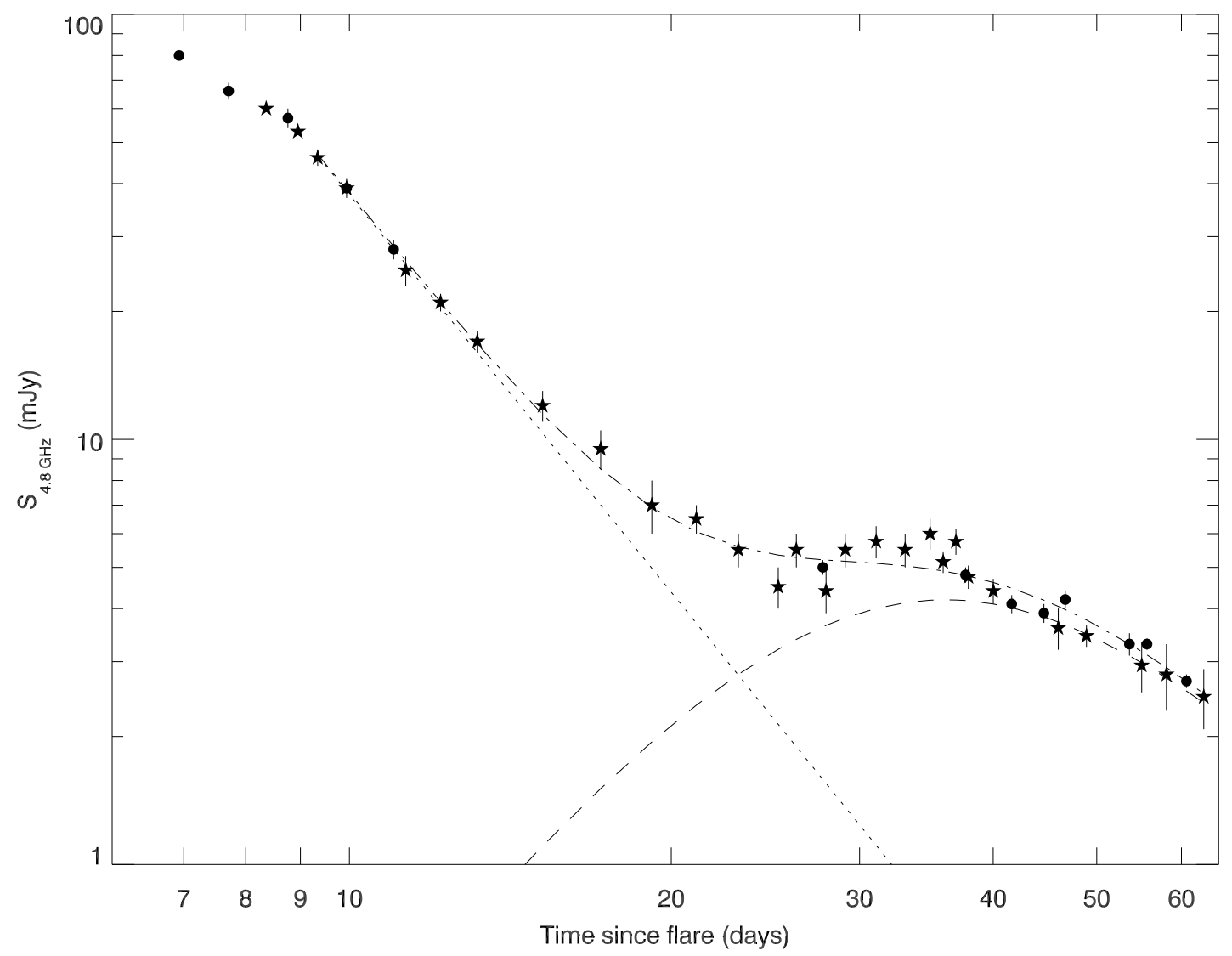

FIG. 1. - The $4.8 \mathrm{GHz}$ light curve of the radio nebula associated with SGR 1806-20. The circles represent data taken with the VLA, and stars data taken with the ATCA. The dot-dashed line in the light curve are the result of fitting the data to the model described in $\$ 3$ and whose parameters are given in the text. The dotted line shows the power-law component of the model fit while the dashed line shows the additional component due to the swept-up, shocked, ambient material. The ends of the dot-dashed line correspond to the first and last data points included in the fit. 


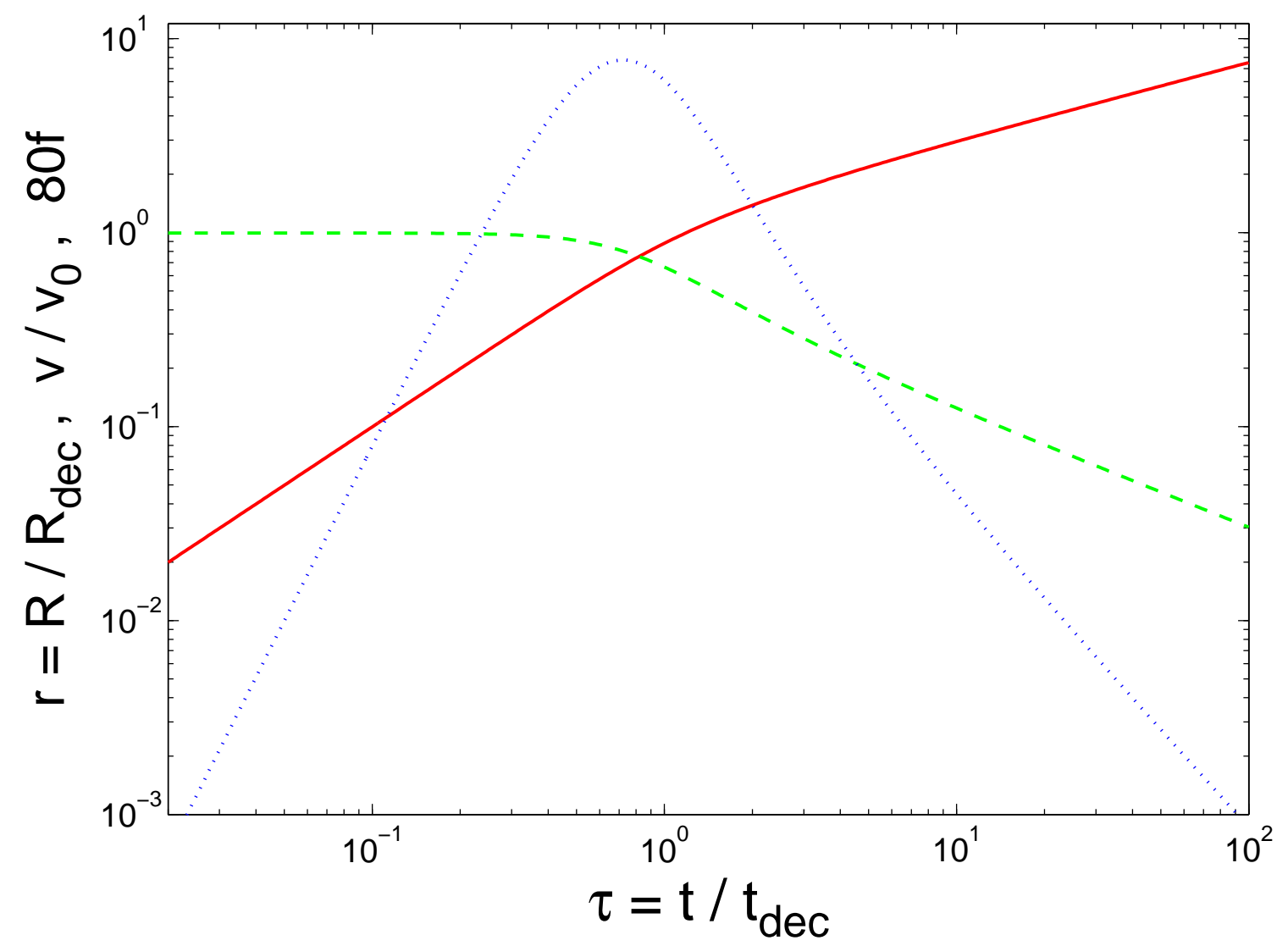

FIG. 2.- Evolution of the expanding shell. The solid red line corresponds to the dimensionless radius, $r$, the dashed green line to the velocity $v$ in units of the initial velocity, and the dotted blue line corresponds to the dimensionless synchrotron flux, $f$. $f$ has been scaled up by a factor of 80 so it falls on the same range as $r$ and $v$. 